\title{
Audiometria de altas frequências no diagnóstico complementar em audiologia: uma revisão da literatura nacional
}

\author{
High-frequency audiometry in audiological complementary \\ diagnosis: a revision of the national literature
}

\author{
Karlin Fabianne Klagenberg ${ }^{1}$, Flávia Cardoso Oliva ${ }^{2}$ Cláudia Giglio de Oliveira Gonçalves ${ }^{3}$, \\ Adriana Bender Moreira Lacerda ${ }^{4}$, Vanessa Gregorczyk Garofani ${ }^{5}$, Bianca Simone Zeigelboim ${ }^{6}$
}

\begin{abstract}
RESUMO
A audiometria de altas frequências (AAF) é um exame audiológico importante na detecção precoce de perdas auditivas por lesões na base do ducto coclear. Nos últimos anos, a sua utilização foi facilitada pelo fato de os audiômetros comercializados passarem a incorporar frequências superiores a $8 \mathrm{kHz}$. Porém, existem diferenças relacionadas aos equipamentos utilizados, às metodologias empregadas e/ou aos resultados e interpretação. Assim, o objetivo deste artigo foi analisar a produção científica nacional sobre a aplicação clínica com AAF, para compreender sua utilização atual. Foram pesquisados textos publicados e indexados nas bases de dados LILACS, SciELO e Medline, num período de tempo de dez anos, utilizando como descritor "audiometria de altas frequências/high-frequency audiometry". Encontraram-se 24 artigos científicos nacionais utilizando AAF, cuja população avaliada, em sua maioria, apresentava de 18 a 50 anos de idade; 13 dos estudos determinaram os limiares utilizando como referência decibel nível de audição (dBNA); alguns estudos realizaram a comparação dos limiares auditivos tonais entre grupos para definir a normalidade; os autores relataram diferenças significativas nos limiares auditivos de altas frequências entre as idades. A AAF é utilizada na clínica audiológica para identificação precoce de alterações auditivas e no acompanhamento da audição de sujeitos expostos a drogas ototóxicas e/ou agentes otoagressores.
\end{abstract}

Descritores: Audição; Audiometria; Audiometria de tons puros; Perda auditiva/diagnóstico; Ducto coclear/lesões

\section{INTRODUÇÃO}

A audiometria de altas frequências (AAF) vem sendo cada vez mais utilizada na prática audiológica ${ }^{(1)}$. Trata-se da avaliação dos limiares auditivos tonais aéreos na faixa de 9

Trabalho realizado no Programa de Mestrado e Doutorado em Distúrbios da Comunicação, Universidade Tuiuti o Paraná - UTP - Curitiba (PR), Brasil. (1) Programa de Pós-graduação em Distúrbios da Comunicação, Universidade Tuiuti do Paraná - UTP - Curitiba (PR), Brasil.

(2) Programa de Pós-graduação em Distúrbios da Comunicação, Universidade Tuiuti do Paraná - UTP - Curitiba (PR), Brasil.

(3) Programa de Pós-graduação em Distúrbios da Comunicação, Universidade Tuiuti do Paraná - UTP - Curitiba (PR), Brasil.

(4) Programa de Pós-graduação em Distúrbios da Comunicação, Universidade Tuiuti do Paraná - UTP - Curitiba (PR), Brasil.

(5) Curso de Especialização em Audiologia Clínica, Universidade Tuiuti do Paraná - UTP - Curitiba (PR), Brasil.

(6) Programa de Pós-graduação em Distúrbios da Comunicação, Universidade Tuiuti do Paraná - UTP - Curitiba (PR), Brasil.

Endereço para correspondência: Karlin Fabianne Klagenberg. R. Acyr Guimarães, 436, cj 1003, Água Verde, Curitiba (PR), Brasil, CEP: 80240-230.

E-mail:karlinfk@yahoo.com.br

Recebido em: 1/12/2009; Aceito em: 10/11/2010
$\mathrm{kHz}$ a $20 \mathrm{kHz}$, sendo um exame complementar importante na detecção precoce de perdas auditivas por lesão na base do ducto coclear. Portanto, sua utilização permite a identificação de alterações auditivas antes que aconteça a perda auditiva na faixa de frequências convencionalmente avaliadas, de $250 \mathrm{~Hz}$ a $8 \mathrm{kHz}^{(2,3)}$.

A pesquisa dos limiares das altas frequências fornece informações importantes sobre a decodificação dos sinais de fala, isso porque as altas frequências são necessárias para a discriminação dos sons consonantais e reconhecimento de fala, e pessoas que possuem algum déficit auditivo nessas frequências apresentam dificuldades de compreensão de fala em ambientes ruidosos ${ }^{(4)}$. A aplicação primária da AAF ocorre no monitoramento da audição de indivíduos sob risco de desenvolverem alterações auditivas causadas por fatores exógenos ou endógenos, como na detecção precoce de perdas auditivas induzidas por drogas ototóxicas, por exposição ao ruído ou por outros processos degenerativos no órgão espiral ${ }^{(1,5-9)}$. Além disso, permite o monitoramento da audição de pacientes com presença ou suspeita de outras doenças que comprometem a audição, como: otite média e suas sequelas, presbiacusia, 
insuficiência renal, distúrbios de processamento auditivo e deficiência auditiva de origem genética ${ }^{(10-12)}$.

Pesquisas científicas utilizando AAF têm sido realizadas desde 1929, sendo Fletcher um dos pioneiros; foi introduzida clinicamente no início da década de 60 , pois estudos anteriores mostravam que danos na audição não poderiam ser detectados apenas pela audiometria convencional $(250 \mathrm{~Hz} \text { a } 8 \mathrm{kHz})^{(1)}$. A utilização da AAF foi facilitada nos últimos anos, pelo fato de os audiômetros comercializados passarem a incorporar frequências superiores a $8 \mathrm{kHz}^{(9,11)}$.

Diversos estudos alertam para o fato de que ainda não existe um consenso com relação aos padrões de calibração das altas frequências. Ocorrem diferenças na calibração dos equipamentos utilizados, nas metodologias empregadas, nos resultados encontrados e na sua interpretação, de acordo com a população estudada ${ }^{(5,8,13)}$. Diante do exposto, o objetivo do presente estudo foi analisar a produção científica nacional a respeito da aplicação clínica da AAF e sua utilização.

\section{REVISÃO DE LITERATURA}

Estabeleceu-se, como universo de análise, os artigos publicados em revistas nacionais e internacionais indexadas nas bases de dados de reconhecimento internacional para as ciências da saúde, LILACS, SciELO e MEDLINE, pesquisados na Biblioteca Virtual em Saúde, num período de tempo de dez anos, utilizando o termo "audiometria de altas frequências/ high-frequency audiometry".

Foram identificados neste período 47 artigos no total, destes, 24 eram relacionados à literatura nacional. Foram também analisados os principais textos de autores de livros na área de Audiologia que abordaram esse tema e alguns artigos clássicos e significativos publicados antes de $1998^{*}$.

$\mathrm{O}$ acesso aos artigos foi por meio da biblioteca virtual BIREME (www.bireme.br) e, após verificar se o artigo atendia ao critério de inclusão para este estudo, foi solicitado o artigo na íntegra.

$\mathrm{Na}$ análise dos artigos, foram descritos os procedimentos, parâmetros para a execução da AAF, população estudada e resultados encontrados pelos autores. A partir do material levantado, procurou-se delinear o contexto da discussão desse estudo e apontar possíveis aplicações e procedimentos.

A revisão da literatura foi dividida em tópicos: aplicações clínicas, variáveis e padrões de exame na AAF.

No levantamento realizado na BIREME, entre 1998 a 2008, encontrou-se 24 artigos científicos nacionais utilizando AAF, em sua maioria ( $45 \%$ ) a população avaliada apresentava de 18 a 50 anos de idade; 13 (52\%) dos estudos determinaram os limiares utilizando, como referência, decibel nível de audição (dBNA).

No Quadro 1 são apresentados os 24 estudos/artigos analisados nesta revisão da literatura.
Foram encontrados 12 artigos com normo-ouvintes, três artigos com exposição a ruído, dois artigos com ênfase em zumbido, quatro artigos relacionados ao monitoramento auditivo no uso de ototóxicos (quimioterápicos e aminoglicosídeos), um artigo relacionado ao uso de otoagressores (solvente), um artigo com referência a alteração metabólica (diabete melito) e por último, um artigo referente à alteração de orelha média.

\section{Estudos sobre aplicação clínica das AAF}

Estudos sobre a aplicação clínica da AAF foram realizados por diferentes autores nos últimos dez anos e estão relacionados ao monitoramento audiológico de indivíduos que fizeram uso de drogas potencialmente ototóxicas, dentre elas a cisplatina $^{(3,7,19)}$ e os solventes ${ }^{(18,31)}$, no tratamento de pacientes com insuficiência renal crônica ${ }^{(17)}$, em indivíduos expostos a ruído $^{(6)}$, e como avaliação complementar das perdas auditi$\operatorname{vas}^{(32)}$. No estudo que monitorou a audição de pacientes com câncer, submetidos a tratamento com cisplatina (considerada ototóxica, dependendo de sua dosagem e administração - doses elevadas e únicas são mais agressivas para a audição), foi realizada a audiometria convencional $(250 \mathrm{~Hz}$ a $8 \mathrm{kHz})$ e de altas frequências $(9 \mathrm{kHz}$ a $20 \mathrm{kHz})$ antes e durante o tratamento quimioterápico. Os resultados demonstraram que a alteração auditiva nas altas frequências foi pontual e progressiva, ou seja, após uma ou duas sessões de quimioterapia, $100 \%$ dos pacientes apresentaram piora da audição nas frequências a partir de $9 \mathrm{kHz}^{(3)}$. Em outro estudo ${ }^{(19)}$, que também monitorou a audição de pacientes expostos à cisplatina, avaliando 13 indivíduos por meio da pesquisa dos limiares auditivos nas frequências de $250 \mathrm{~Hz}$ a $18 \mathrm{kHz}$ e teste de emissões otoacústicas transitórias (EOAT) e produto de distorção (EOAPD) observou-se que, após a infusão de $120 \mathrm{mg} / \mathrm{m}^{2}$ de cisplatina, os limiares de audibilidade pioraram a partir da frequência de $8 \mathrm{kHz}$. A resposta global e as amplitudes absolutas nas frequências de 1,2 e $3 \mathrm{kHz}$ da EOAT tendem a permanecer presentes até o final do tratamento, o mesmo não ocorre com a frequência de $4 \mathrm{kHz}$; os autores concluíram que a AAF foi um método mais efetivo na detecção precoce da ototoxidade da cisplatina.

Outro estudo ${ }^{(17)}$ investigou a audição nas altas frequências ( $9 \mathrm{kHz}$ até $18 \mathrm{kHz}$ ), em pacientes com insuficiência renal crônica submetidos a tratamento conservador, no período de um ano, comparados com um grupo controle da mesma faixa etária. Os resultados mostraram um declínio na sensibilidade auditiva com o aumento da frequência em todas as faixas etárias estudadas, principalmente na segunda avaliação, sendo esta mais acentuada ou evidente na faixa etária de 50 a 59 anos. A conclusão do estudo sugere que a AAF é um importante recurso não só para a detecção precoce da perda auditiva, mas de seu monitoramento em pacientes portadores de insuficiência renal crônica.

Os estudos acima citados que utilizaram as AAF para monitoramento auditivo em pacientes durante tratamento com

"Skinner MW, Miller JD. Amplification bandwidth and intelligibility of speech in quiet and noise for listeners with sensorineural hearing loss. Audiology. 1983;22(3):253-79.

Shaw GM, Jardine CA, Fridjhon P. A pilot investigation of high-frequency audiometry in obscure auditory dysfunction (OAD) patients. Br J Audiol. 1996;30(4):233-7.

Hallmo P, Sundby A, Mair IW. High-frequency audiometry. Response characteristics of the KH70 vibrator. Scand Audiol. 1991;20(2):139-43. 
Quadro 1. Síntese dos artigos nacionais com AAF, nos últimos dez anos $(n=24)$

\begin{tabular}{|c|c|c|c|c|c|}
\hline Ano & Autores & $\begin{array}{l}\text { Total de } \\
\text { sujeitos }\end{array}$ & $\begin{array}{l}\text { Faixa etária } \\
\quad(\text { anos })\end{array}$ & $\begin{array}{l}\text { Característica auditiva da população } \\
\text { estudada }\end{array}$ & Referência \\
\hline 1999 & Azevedo e lorio(14) & 52 & 12 a 15 & Sujeitos com audição normal até $8000 \mathrm{~Hz}$ & NPS \\
\hline 2000 & Beltrami $^{(15)}$ & 101 & 25 a 35 & Adultos expostos e não expostos ao ruído & NPS \\
\hline 2000 & $\begin{array}{l}\text { Pedalini, Sanchez, D’Antonio, D'Antonio, } \\
\text { Balbani, Hachya, Liberman, Bento(16) }\end{array}$ & 158 & 4 a 60 & Sujeitos com audição normal até $8000 \mathrm{~Hz}$ & NA \\
\hline 2001 & $\begin{array}{l}\text { Zeigelboim, Mangabeira-Albernaz e } \\
\text { Fukuda }^{(17)}\end{array}$ & 133 & 30 a 59 & $\begin{array}{l}\text { Sujeitos com audição normal até } 8000 \mathrm{~Hz} \text { e } \\
\text { grupo com insuficiência renal crônica }\end{array}$ & NPS \\
\hline 2001 & Fernandes e Mota ${ }^{(18)}$ & 25 & 17 a 34 & Trabalhadores expostos a ruído e solventes & NPS \\
\hline 2003 & Garcia, lório, Petrilli(19) & 13 & 7 a 20 & $\begin{array}{l}\text { Sujeitos com osteossarcoma expostos a } \\
\text { cisplatina }\end{array}$ & NPS \\
\hline 2003 & Sahyeb, Costa e Alvarenga ${ }^{(13)}$ & 50 & 18 a 30 & jovens com audição normal até $8000 \mathrm{~Hz}$ & NA \\
\hline 2004 & $\begin{array}{l}\text { Castro, Conde, Paiva, Oliveira, } \\
\text { Bernardi }^{(20)}\end{array}$ & 60 & 18 a 49 & Trabalhadores expostos a ruído & NPS \\
\hline 2004 & Burguetti, Peloggia, Carvallol(21) & 71 & 20 a 60 & Sujeitos com queixa de zumbido & NA \\
\hline 2004 & $\begin{array}{l}\text { Retamal, Marochi, Ziegelboim, } \\
\text { Marques }^{(22)}\end{array}$ & 45 & 12 a 19 & jovens com audição normal até $8000 \mathrm{~Hz}$ & NPS \\
\hline 2004 & Porto, Gahyva, Lauris, Lopes(23) & 60 & 21 a 60 & Adultos expostos e não expostos ao ruído & NA \\
\hline 2004 & $\begin{array}{l}\text { Zeigelboim, Oliveira, Marques, } \\
\text { Jurkievcz }^{(24)}\end{array}$ & 30 & 20 a 30 & Sujeitos com audição normal até $8000 \mathrm{~Hz}$ & NA \\
\hline 2005 & Ramos e Pereira ${ }^{(10)}$ & 32 & 9 a 10 & escolares & NPS \\
\hline 2005 & Martinho, Zeigelboim e Marques(25) & 66 & 30 a 40 & Sujeitos com audição normal até $8000 \mathrm{~Hz}$ & NPS \\
\hline 2005 & $\begin{array}{l}\text { Jacob, Stumpf, Bitencourt, Marques, } \\
\text { Puppi, Gonçalves }{ }^{(8)}\end{array}$ & 44 & 3 a 28 & Sujeitos expostos a agentes quimioterápicos & NA \\
\hline 2006 & Almeida e Nishimori(26) & 55 & 18 a 25 & Sujeitos com audição normal até $8000 \mathrm{~Hz}$ & NA \\
\hline 2006 & Silva e Feitosa ${ }^{(27)}$ & 64 & $\begin{array}{l}25 \text { a } 35 \text { e } \\
45 \text { a } 55\end{array}$ & Sujeitos com audição normal até $8000 \mathrm{~Hz}$ & NA \\
\hline 2006 & Reis, Tschiedel e Broto ${ }^{(28)}$ & 61 & Sem dado & Grupo controle e grupo com diabetes tipo I & Sem dado \\
\hline 2007 & Carvallo, Koga, Carvalho e Ishida ${ }^{(11)}$ & 74 & 18 a 30 & Sujeitos com audição normal até $8000 \mathrm{~Hz}$ & NA \\
\hline 2007 & $\begin{array}{l}\text { Sá, Lima, Tonita, Frota, Sants, } \\
\text { Garcia(29) }^{(29)}\end{array}$ & 51 & 18 a 29 & Sujeitos com audição normal até $8000 \mathrm{~Hz}$ & NA \\
\hline 2007 & Figuerêdo e Corona(9) & 30 & 19 a 56 & $\begin{array}{l}\text { Sujeitos com audição normal até } 8000 \mathrm{~Hz} \\
\text { com e sem presença de zumbido }\end{array}$ & NA \\
\hline 2007 & Ferreira, Almeida e Atherino(12) & 31 & 7 a 12 & $\begin{array}{l}\text { Crianças com audição normal com } \\
\text { episódios de otite média secretora bilateral }\end{array}$ & NA \\
\hline 2007 & Lopes, Almeida, Zanconato, Mondelli(30) & 30 & 7 a 13 & Crianças com audição normal até $8000 \mathrm{~Hz}$ & NPS \\
\hline 2008 & Almeida, Umeoka, Viera, Moraes ${ }^{(3)}$ & 10 & 5 a 27 & Sujeitos tratados com cisplatina & NA \\
\hline
\end{tabular}

Legenda: NA = nível de audição; NPS = nível de pressão sonora

medicamentos ototóxicos (quimioterápicos ou conservadores da função renal), visando prevenir as alterações auditivas permanentes, reforçam a importância da aplicabilidade clinica do teste para este fim.

No estudo que monitorou a audição de indivíduos expostos a ruído, os autores ${ }^{(18)}$ encontraram um decréscimo dos limiares auditivos tonais nas altas frequências em ambas as orelhas conforme o aumento das altas frequências, sendo mais acentuada a partir de 14kHz. Em ambas as orelhas houve diferença estatística entre os limiares obtidos nas frequências de 10 e 16 kHz, 10 e 18 kHz, 12 e 16 kHz, 12 e 18 kHz, 14 e 16 kHz e 8 $\mathrm{kHZ}$ em trabalhadores expostos a ruído e solventes. Também foi verificada piora dos limiares de audibilidade médios de $\mathrm{AAF}$ dos indivíduos expostos a ruído e solvente, quando comparados aos demais estudos de padronização de limiares de normo-ouvintes.
Estudos realizados em indivíduos expostos a ruído ocupacional e não expostos, que apresentaram limiares auditivos dentro dos padrões aceitáveis nas frequências de $250 \mathrm{~Hz}$ a $8 \mathrm{kHz}$, mostraram diferenças nos limiares auditivos de altas frequências em relação aos grupos, sendo que, no grupo de indivíduos não expostos ao ruído, os limiares auditivos foram melhores, sugerindo que a avaliação das altas frequências, acima de $8 \mathrm{kHz}$, pode fornecer indícios da lesão por ruído comparativamente à audiometria convencional ${ }^{(20,34)}$.

Estes estudos demonstram que a AAF é sensível para detectar alteração auditiva precoce nos indivíduos expostos a ruído e ou agentes químicos e são recomendados como avaliação auditiva complementar nos programas de prevenção de perda auditiva de origem ocupacional.

Quanto à avaliação complementar visando o diagnóstico 
das perdas auditivas, um estudo ${ }^{(32)}$ sugere que sujeitos com perda auditiva neurossensorial de grau severo e profundo, bilateral, pré-lingual, com presença de respostas para tons de alta frequência, tem melhor qualidade de fala quando comparados aos sujeitos com ausência de respostas nas AAF. Outro estudo $^{(34)}$ já revelou que as altas frequências são importantes para a discriminação dos sons consonantais e o reconhecimento de fala, e que pessoas com perda auditiva nessas frequências teriam dificuldade em destacar o sinal do ruído, apresentando alteração de compreensão em ambientes ruidosos. Uma vez que as altas frequências são importantes na compreensão da fala, a utilização da AAF contribuiria com informações para a análise do processamento auditivo central ${ }^{(10)}$.

Os estudos que usaram as AAF para fins de diagnóstico das perdas auditivas revelaram um dado importante no que se refere à relação das respostas auditivas para as altas frequências com a compreensão e a qualidade de fala, contribuindo desta maneira para a prevenção, diagnóstico e tratamento das alterações de fala e de processamento auditivo central.

\section{Variáveis consideradas na avaliação de sujeitos por AAF}

Considerações sobre as variáveis: gênero, idade, orelha testada e dificuldades na audibilização de tons em altas frequências foram relatadas na literatura, alertando para sua influência na avaliação auditiva nas altas frequências.

Em relação ao gênero, estudos apontam que, frequentemente, as mulheres têm melhor sensibilidade auditiva do que os homens para sons de alta frequência, porém, em se tratando de uma população jovem e audiologicamente normal, os resultados das pesquisas geralmente não têm demonstrado diferenças significativas nessa comparação ${ }^{(13,30)}$.

Quanto à idade, o processo de envelhecimento auditivo envolve a perda de sensibilidade auditiva para altas frequências. Por meio desta é possível perceber o desgaste auditivo causado pelo fator idade antes mesmo de aparecerem sinais na audiometria convencional ${ }^{(16,28,35)}$. Em outro estudo ${ }^{(36)}$ americano, em 2003, os autores alertaram que crianças muito pequenas não possuem bom desempenho na avaliação auditiva por AAF, e o início da perda auditiva nas mesmas poderá ser observado por volta dos dez anos de idade, porém nessa idade há melhor sensibilidade auditiva acima de $14 \mathrm{kHz}$.

Foram relatadas em um estudo ${ }^{(32)}$ diferenças significativas entre as médias tonais entre as orelhas direita e esquerda para as frequências de $9 \mathrm{kHz}$ e $11,2 \mathrm{kHz}$ de acordo com a idade. Conforme o aumento da frequência e também da idade, a diferença permanecia entre 10 e $20 \mathrm{~dB}$.

Mais um estudo ${ }^{(37)}$ enfatiza também a necessidade de se controlar a variável idade na investigação de sujeitos expostos ao ruído quando se realiza a AAF, pois a idade interfere nos limiares a partir de $8 \mathrm{kHz}$ mais do que interfere nos limiares até $8 \mathrm{kHz}$, nos quais a ação do ruído é predominante a da idade.

Em relação à dominância auditiva, vários estudos realizados demonstraram concordância de resultados no que diz respeito à ausência de diferenças entre os limiares de altas frequências obtidos nas orelhas esquerda e direita ${ }^{(9,11,13,16,17,22)}$. Porém, um deles ${ }^{(26)}$ indicou melhor limiar auditivo para a orelha esquerda, explicada provavelmente pelo fato de as avaliações auditivas terem sido iniciadas sempre pelo lado direito, o que teria beneficiado o lado esquerdo pelo efeito de treinamento auditivo após a primeira avaliação.

A avaliação de tons puros em altas frequências pode ser comprometida por se tratarem de tons não familiares à maioria dos indivíduos, o que afetaria a confiabilidade das suas respostas. Porém, para controlar esse provável fator, um estudo ${ }^{(7)}$ testou as altas frequências nos mesmos indivíduos em dias consecutivos, não encontrando diferenças significativas nos limiares auditivos tonais intra-sujeitos nas diversas avaliações.

\section{Padrões técnicos utilizados nas AAF}

Diversos fatores na execução do exame de AAF devem ser considerados, pois podem levar a variações dos resultados, tais como: treinamento prévio do sujeito na realização do exame, tipo de audiômetro utilizado para a avaliação auditiva de tons de alta frequência, calibração do equipamento segundo referência do decibel em nível de audição (dBNA) ou em nível de pressão sonora (dBNPS) e o posicionamento do fone no ouvido, que pode interferir na precisão do exame devido às características de ressonância do meato acústico externo para sinais acima de $15 \mathrm{kHz}$, podendo ocorrer variações de 15 a $20 \mathrm{~dB}$ na resposta do sujeito; também poderá ocorrer maior possibilidade de efeito do colabamento do meato acústico externo, principalmente na avaliação das altas frequências ${ }^{(13,27)}$.

Não há um padrão de normalidade definido para as altas frequências; portanto, os autores recomendaram a comparação dos limiares auditivos tonais entre grupos, controlando principalmente a variável idade e definindo padrões semelhantes entre os grupos para os limiares tonais convencionais ${ }^{(1,38)}$.

Outra questão relatada refere-se a melhoras dos limiares auditivos nas altas frequências depois de procedimentos otocirúrgicos (principalmente na otoesclerose), mas salientam que não está clara a natureza dessas melhoras nos limiares, uma vez que a avaliação dos limiares tonais aéreos por via óssea se restringe a $6 \mathrm{kHz}$ e, mesmo com a possibilidade da avaliação por via óssea nas altas frequências $(8 \mathrm{kHz}$ a $16 \mathrm{kHz})$, essa técnica ainda está em fase de pesquisa experimental, sem aplicabilidade na prática clínica ${ }^{(39)}$.

\section{DISCUSSÃO}

Observaram-se diversos consensos com relação à importância da AAF nos estudos relatados. Um deles refere-se ao monitoramento auditivo e à detecção precoce de perdas auditivas por otoxidade, principalmente as induzidas pela cisplatina, que apresenta claramente o decréscimo das AAF durante o tratamento, podendo ser uma ferramenta muito importante para definir a necessidade de mudança da droga por recomendação médica ${ }^{(3,19)}$. Destaca-se que o monitoramento não sofre influência da idade e da calibração do equipamento, uma vez que para a sua realização é utilizada como ponto de partida a audiometria inicial, antes do tratamento, comparando-a às audiometrias posteriores.

A AAF é um bom método de investigação clínica, podendo integrar o protocolo de investigação audiológica inicial em casos de exposição a agentes otoagressores (ruído e produtos 
químicos), ototoxicidade e doenças sistêmicas que podem afetar o sistema auditivo, conforme diversos autores ${ }^{(3,6,7,17-19,31,32)}$.

Uma grande parte dos estudos realizados utilizou populações jovens e audiologicamente normais, comparando os grupos para identificar as diferenças nos limiares auditivos, uma vez que não existe uma padronização de normalidade para as altas frequências ${ }^{(9,11,13,14,16,17,22,24-27,29)}$.

Os autores relataram diferenças significativas nos limiares auditivos entre as idades, sendo que nos estudos analisados há referência de piora gradual da sensibilidade auditiva para as altas frequências com o avanço da idade ${ }^{(27)}$.

Há referências sobre os cuidados durante a execução do exame de AAF, por exemplo, em relação ao posicionamento do fone e ao treinamento prévio, os autores concordam quanto à importância do cuidado antes e durante a realização do exame, para que não exista alteração nas respostas dos sujeitos.

Em relação à utilização da medida em decibel nível de audição (NA) ou nível de pressão sonora (NPS), observou-se um uso bastante variável na literatura pesquisada. Uma vez que a audiometria convencional utiliza o decibel NA, torna-se mais compreensível para efeitos de análise e comparação a utilização do decibel NA nas altas frequências, porém alguns equipamentos só utilizam o decibel NPS, devido à sua calibração mais precisa, o que implica na conversão para decibel NA caso se opte por sua utilização. Para a conversão dos valores, há necessidade de observarem-se alguns cuidados, conforme orientações dos fabricantes dos audiômetros; contudo, não foi encontrado nenhum artigo que referenciasse esse aspecto.

\section{COMENTÁRIOS FINAIS}

A AAF vem sendo amplamente utilizada na prática audiológica, para a identificação precoce de alterações auditivas, no monitoramento auditivo de sujeitos expostos a drogas ototóxicas ou a agentes otoagressores e no diagnóstico complementar quando se avaliam populações especiais como no caso dos portadores de doenças renais crônicas, diabetes e patologias auditivas. Há consenso quanto aos cuidados na execução do exame e à idade do sujeito nos limiares auditivos em altas frequências. Sugerimos estudos epidemiológicos visando à determinação de um padrão nacional de normatização para indivíduos normo-ouvintes para a AAF.

\begin{abstract}
High-frequency audiometry (HFA) is an important audiological test for early detection of hearing losses caused by leasions in the base of the cochlear duct. In recent years, its use was facilitated because audiometers began to identify frequencies higher than $8 \mathrm{kHz}$. However, there are differences related to the equipment used, the methodologies followed, and/or to the results and their interpretation. Therefore, the aim of this study was to analyze the national scientific production regarding HFA clinical application, to better understand its current use. Articles published and indexed in LILACS, SciELO and Medline databases in the last ten years were researched, using as keyword "audiometria de alta frequência/high-frequency audiometry". Twenty-four Brazilian scientific papers using HFA were found; most studies evaluated participants with ages ranging from 18 to 50 years; 13 studies used decibel hearing level (dBHL) to determine the thresholds; a few studies compared tonal auditory thresholds between groups to define normality; the authors reported significant differences in high frequency auditory thresholds among age ranges. HFA is used in audiological clinic for early identification of auditory alterations and in the auditory follow-up of subjects exposed to ototoxic drugs and/or otoagressive agents.
\end{abstract}

Keywords: Hearing; Audiometry; Audiometry, pure-tone; Hearing loss/diagnosis; Cochlear duct/injuries

\section{REFERÊNCIAS}

1. Zeigelboim BS, Fukuda Y, Iório MCM. Audiometria de alta frequência. Acta AWHO. 1996;15(3):155-8.

2. Reis HG, Garcia DC, Martin-Polo T, Moussalle SK. Utilidades clínicas da audiometria de altas frequências. Rev Med PUCRS. 2002;12(2):1746.

3. Almeida EOC, Umeoka WG, Viera RC, Moraes IF. Estudo audiométrico de alta frequência em pacientes curados de câncer tratados com cisplatina. Rev Bras Otorrinolaringol. 2008;74(3):382-90.

4. Rissatto MR, Santos TMM. Limiares tonais em frequências ultra-altas e reconhecimento de fala de portadores de perda auditiva neurossensorial. Rev CEFAC. 2003;5(3):279-83.

5. Feghali JG, Bernstein RS. A new approach to serial monitoring of ultrahigh frequency hearing. Laryngoscope. 1991;101(8):825-9.

6. Hallmo P, Borchgrevink HM, Mair IW. Extended high-frequency thresholds in noise-induced hearing loss. Scand Audiol. 1995;24(1):4752 .

7. Fausti SA, Henry JA, Hayden D, Phillips DS, Frey RH. Intrasubject reability of high-frequency $(9-14 \mathrm{kHz})$ thresholds: tested separately vs. following conventional-frequency testing. J Am Acad Audiol. 1998;9(2):147-52.

8. Jacob LCB, Stumpf CC, Bitencourt RF, Marques JM, Puppi C, Gonçalves PT. Avaliação audiológica em indivíduos com neoplasias expostos a agentes quimioterápicos. Fono Atual. 2005;8(31):12-25.

9. Figuerêdo RBS, Corona AP. Influência do zumbido nos limiares auditivos de altas frequências. Rev Soc Bras Fonoaudiol. 2007;12(1):29-33.

10. Ramos CS, Pereira LD. Processamento auditivo e audiometria de altas frequências em escolares de São Paulo. Pró-Fono. 2005;17(2):153-64.

11. Carvallo RMM, Koga MC, Carvalho M, Ishida IM. Limiares auditivos para altas frequências em adultos sem queixa auditiva. Acta ORL. 2007;25(1):62-6.

12. Ferreira MS, Almeida K, Atherino CCT. Limiares de audibilidade em altas frequências em crianças com história de otite média secretora bilateral. Rev Bras Otorrinolaringol. 2007;73(2):231-8.

13. Sahyeb DR, Costa Filho OA, Alvarenga KF. Audimetria de alta frequência: estudo com indivíduos audiologicamente normais. Rev Bras Otorrinolaringol. 2003;69(1):93-9. 
14. Azevedo LL, Iório MCM. Estudo dos limiares de audibilidade nas altas frequências em indivíduos de 12 a 15 anos com audição normal. Acta AWHO. 1999;18(2):78-85.

15. Beltrani CHB. Dos limiares de audibilidade nas frequências de 250 a $18.000 \mathrm{~Hz}$ em indivíduos expostos a ruído ocupacional [tese]. São Paulo: Universidade Federal de São Paulo. Departamento de Fonoaudiologia; 1999.

16. Pedalini MEB, Sanchez TG, D'Antonio A, D'Antonio W, Balbani A, Hachiya A, et al. Média dos limiares tonais na audiometria de alta frequência em indivíduos normais de 4 a 60 anos. Pró-Fono. 2000;12(2):17-20.

17. Zeigelboim BS, Mangabeira-Albernaz PL, Fukuda Y. High frequency audiometry and chronic renal failure. Acta Otolaryngol. 2001;121(2):245-8.

18. Fernandes JB, Mota HB Estudo dos limiares de audibilidade nas altas frequências em trabalhadores expostos a ruído e solvente. Pró-Fono. 2001;13(1):1-8.

19. Garcia AP, Iório MCM, Petrilli AS. Monitoramento da audição de pacientes expostos à cisplatina. Rev Bras Otorrinolaringol. 2003;69(2):215-21.

20. Castro IFC, Conde CA, Paiva APQF, Oliveira LTN, Bernardi APA. Estudo do perfil audiométrico em alta frequência em trabalhadores expostos a ruído. Rev CEFAC. 2004;6(2):203-8.

21. Burguetti FAR, Peloggia AG, Carvallo RMM. Limiares de audibilidade em altas frequências em indivíduos com queixa de zumbido. Arq Int Otorrinolaringol. 2004;8(4):277-83.

22. Retamal MCR, Marochi R, Zeigelboim BS, Marques JM. Estudo dos limiares de audibilidade nas altas frequências em indivíduos monitoramento normo-ouvintes de 12 a 19 anos. Distúrb Comun. 2004;16(1):35-42.

23. Porto MAA, Gahyva DLC, Lauris JRP, Lopes AC. Avaliação da audição em frequências ultra-altas em indivíduos expostos ao ruído ocupacional. Pró-Fono. 2004;16(3):237-50.

24. Zeigelboim BS, Oliveira VFBG, Marques JM, Jurkiewicz AL. Limiares de audibilidade nas altas frequências em indivíduos de 20 a 30 anos com audição normal. Distúrb Comun. 2004;16(3):385-92.

25. Martinho T, Zeigelboim BS, Marques JM. Perfil audiológico nas frequências em indivíduos de 30 a 40 anos com audição normal. Arq Int Otorrinolaringol. 2005;9(1):18-25.

26. Almeida EOC, Nishimori AY. Influência do posicionamento do fone na audiometria de alta frequência. Rev Bras Otorrinolaringol. 2006;72(5):691-8.
27. Silva IMC, Feitosa MAG. Audiometria de alta frequência em adultos jovens e mais velhos quando a audiometria convencional é normal. Rev Bras Otorrinolaringol. 2006;72(5):665-72.

28. Reis HG, Tschiedel B, Broto JP. Limiares audiométricos de altas frequências em pacientes com diabetes mellitus insulino-dependente. Sci Med. 2006;16(1):16-22.

29. Sá LCB, Lima MAMT, Tomita S, Frota SMMC, Santos GA, Garcia TR. Avaliação dos limiares de audibilidade das altas frequências em indivíduos entre 18 e 29 anos sem queixas otológicas. Rev Bras Otorrinolaringol. 2007;73(2):215-25.

30. Lopes AC, Almeida BK, Zanconato CM, Mondelli MFCG. Estudo dos limiares de audibilidade de altas frequências em crianças ouvintes com idade entre 7 e 13 anos. Distúrb Comun. 2007;19(2):173-80.

31. Fuente A, McPherson B. Organic solvents and hearing loss: The challenge for audiology. Int J Audiol. 2006;45(7):367-81.

32. Kotzias SA. Influência na fala das altas frequências em portadores de hipoacusia neurossensorial severa e profunda bilateral pré-lingual [tese]. São Paulo: Faculdade de Medicina da Universidade de São Paulo; 2004.

33. Somma G, Coppeta L, Magrini A, Parrella M, Cappelletti MC, Gardi S, Messina M, Bergamaschi A; Italcementi Group Bergamo. [Extended high frequency audiometry in the prevention of noise-induced hearing loss]. G Ital Med Lav Ergon. 2007;29(3 Suppl):258-60. Italian.

34. Mets JT. Re: Ahmed HO, Dennis JH, Badran O, et al. High frequency $(10-18 \mathrm{kHz})$ hearing thresholds: reliability, and effects of age and occupational noise exposure. Occup Med 2001; 51: 245-258. Occup Med (Lond). 2001;51(8):519-22.

35. Skinner MW, Miller JD. Amplification bandwidth and intelligibility of speech in quiet and noise for listeners with sensorineural hearing loss. Audiology. 1983;22(3):253-79.

36. Dubno JR, Horwitz AR, Ahlstron JB. Recovery from prior stimulation: masking of speech by interrupted noise for younger and older adults with normal hearing. J Acoust Soc Am. 2003;111(4 Pt 1):2084-94. Comment on: J Acoust Soc Am. 2002;111(6):2897-907.

37. Schochat E, Rabelo CM, Sanfins MD. Processamento auditivo central: testes tonais de padrão de frequência e de duração em indivíduos normais de 7 a 16 anos de idade. Pró-Fono. 2000;12(2):1-7.

38. Shaw GM, Jardine CA, Fridjhon P. A pilot investigation of highfrequency audiometry in obscure auditory dysfunction (OAD) patients. Br J Audiol.1996;30(4):233-7.

39. Hallmo P, Sundby A, Mair IW. High-frequency audiometry. Response characteristics of the KH70 vibrator. Scand Audiol. 1991;20(2):139-43. 